\title{
A qualitative exploration of the experiences of primary care patients engaged in email counseling meant to increase physical activity
}

\author{
Tomas Vetrovsky ${ }^{1, *}$, Klaudia Vetrovska ${ }^{2}$, and Vaclav Bunc ${ }^{1}$ \\ ${ }^{1}$ Faculty of Physical Education and Sport, Charles University, Prague, Czech Republic; and ${ }^{2}$ Humilitas s.r.o., Beroun, \\ Czech Republic
}

Copyright: (C) 2019 T. Vetrovsky et al. This is an open access article licensed under the Creative Commons Attribution License (https://creativecommons.org/licenses/by/4.0/).

\begin{abstract}
Background: As insufficient physical activity is a widespread public health problem, the patients' perspective and experiences during their efforts to increase levels of physical activity need to be explored and elucidated. Objectives: The purpose of our study was to qualitatively analyze the patient-written email messages in order to explore patients' experiences during a 12-week pedometer-based physical activity intervention in a primary care setting that was paired with email counseling. Methods: The content of 31 email messages from 10 participants was extracted, coded, and analyzed using thematic analysis. Results: We identified 22 themes that were grouped into 3 categories: reflections on the pedometer-based intervention, use of behavior change techniques, and barriers that affected participants' engagement in physical activity. We have also anecdotally described several interesting aspects of behavior change techniques: negative attitudes elicited by the goal setting process, learning from their own data, and enjoyment associated with self-monitoring. Conclusion: Qualitatively analyzing email messages written as part of the physical activity counseling process is useful in documenting patients' perceptions and use of different behavior change techniques and their reported barriers that get in the way of increasing their physical activity.
\end{abstract}

Keywords: qualitative research, thematic analysis, email counseling, pedometer; physical activity, behavior change techniques

\section{Introduction}

Sedentary lifestyles and physical inactivity are major health problems that increase the incidence of numerous chronic diseases and premature death (Pedersen \& Saltin, 2015; Reiner, Niermann, Jekauc, \& Woll, 2013; Warburton, Nicol, \& Bredin, 2006; Woodcock, Franco, Orsini, \& Roberts, 2011). As such, establishing effective interventions that increase physical activity (PA) levels are desperately needed. As diverse populations regularly visit their general practitioners, primary care has been shown to be a favorable setting for PA interventions (Gagliardi, Abdallah, Faulkner, Ciliska, \& Hicks, 2015; Hébert, Caughy, \& Shuval, 2012; Jimmy \& Martin, 2005; Orrow, Kinmonth, Sanderson, \& Sutton, 2012). Specifically, many small trials suggest that the use of pedometers and/or individual counseling have the potential to increase habitual PA of primary

\footnotetext{
* Address for correspondence: Tomas Vetrovsky, Department of Physiology and Biochemistry, Faculty of Physical Education and Sport, Charles University, José Martího 31, 162 52, Prague 6, Czech Republic. E-mail: tomas.vetrovsky@gmail.com
}

care patients (Gao et al., 2016; Glynn et al., 2014; James et al., 2017; Pears et al., 2016; Richards \& Cai, 2016; van der Weegen et al., 2015). However, although several recent larger trials in primary care confirm the positive effects of pedometer-based PA counseling, these studies also show that the effectiveness of these interventions is far from optimal (Harris et al., 2015), especially in the long term (Harris et al., 2017; Yates et al., 2017). Therefore, the factors that influence patients' effort to increase and maintain their PA need to be explored and elucidated.

Recently, several groups have studied the beliefs, attitudes, behaviors, and experiences of patients participating in PA interventions delivered in a primary care setting (Casey et al., 2014; Wahlich et al., 2017) as well as barriers and facilitators that play a role in changing PA levels (Normansell et al., 2014; Patel, Schofield, Kolt, $\&$ Keogh, 2013). Most of these studies used qualitative analysis of interviews with the participants performed after the interventions were completed (Casey et al., 2014; Normansell et al., 2014; Wahlich et al., 2017). While this is a valid and useful approach, it also has its disadvantages as the interviewees might be influenced 
by the interview situation, and as such, they may not provide reliable information because of either an unconscious bias or even conscious and deliberate attempts to mislead the interviewer (Diefenbach, 2008). Thus, supplementing the interviews with the direct analysis of counseling content can yield additional insight.

Unfortunately, papers that qualitatively analyze the content of PA counseling in primary care are rare (Bardach \& Schoenberg, 2014; Carroll, Antognoli, \& Flocke, 2011), which might be explained by the inconvenience of recording face-to-face or telephone counseling sessions. Fortunately, with the advent of emailbased interventions (Hageman et al., 2017; Hatchett, Hallam, \& Ford, 2012), this inconvenience could be overcome as the content of the counseling is automatically stored on an email server. In the past, email has been used to facilitate qualitative interviews (Egan, Chenoweth, \& McAuliffe, 2009; Hershberger \& Kavanaugh, 2017) and several studies analyzed the emails written by patients in various contexts (Chiu, Marziali, Tang, Colantonio, \& Carswell, 2011; van Esch, Cornel, $\&$ Snoek, 2010) but, to the best of our knowledge, the content of patients' emails written as part of the PA counseling process has not been analyzed before.

Therefore, we explored the experiences of primary care patients participating in a walking intervention and analyzed what strategies they used to increase their daily step count. Specifically, we employed thematic analysis on the email messages written by primary care patients during their pedometer-based PA email counseling to determine factors influencing their effort to increase and maintain their PA.

\section{Methods}

\section{Study design}

The qualitative study was conducted alongside a 12-week pedometer-based walking intervention with email counseling in physically inactive adults recruited from four general practices. Details of the intervention design and its outcomes have been reported elsewhere (Vetrovsky et al., 2018). Briefly, the participants were instructed to wear the pedometer daily, check the step count every evening, and gradually increase the daily number of steps up to 10,000 . They were also required to regularly upload data to a website where the data could be viewed online by the counselor. The counselor communicated with the participants once a week during the first 4 weeks of the intervention, and bi-weekly for the remaining 8 weeks. Altogether, the counselor sent eight counseling emails during the 12-week intervention period. The emails were drafted individually, tailored to the specific needs of the participant and the circumstances of their case. Although individualized, the emails always incorporated some common features: (a) encouragement of the participants based on their objectively measured achievement in the previous week; (b) reminder of the benefits of PA for the physical and mental health relevant to the individual participant; (c) discussion of individual behavioral strategies, what works for them, and what does not; and (d) setting of the goal for the upcoming week. Several behavior change techniques (BCTs) were systematically used in the counseling intervention: goal setting, self-monitoring, action planning, and providing feedback. Only some of the emails written by the counselor included explicit questions related to participant's goals, strategies, and barriers, especially at the beginning of the intervention. However, all counselor's emails attempted to encourage participants to share experiences about their effort to increase walking, even though participants were not specifically instructed to answer all emails.

\section{Study participants}

Of 10 participants, 7 were males and 3 were females; their mean age was 44 ( $S D$ 10). Nine of the participants personally drafted their own email responses, whereas one of the males, aged 64, preferred that his daughter living in the same household participate in email communication on his behalf. All participants actively participated in email communication and, on average, they sent 3.1 ( $S D$ 1.3) messages. Details of individual participants, numbers of emails they sent, and average word count of their emails are depicted in Table 1.

\section{Data collection and sample}

All email messages written by participants throughout the course of counseling were saved and stored for later use. This collection included a wide variety of messages in terms of their size, form, and style: well-structured answers to counselor's questions, free elaboration on participants' experiences, communication related to technical issues (e.g., drained pedometer batteries, troubles with uploading of the pedometer data), and brief replies to counselor's emails ("thank you", "OK", etc.). Messages only dealing with technical issues and brief replies were discarded and only messages with content directly related to PA counseling were included. For the purpose of word count calculation, the messages were stripped from introductory and closing phrases. Altogether, 31 email messages with an average word count of 91 ( $S D$ 69) were included in the analysis.

\section{Data analysis}

The content of the email messages was extracted and analyzed using an inductive approach to thematic analysis to identify themes naturally emerging from 
Table 1

Characteristics of individual participants

\begin{tabular}{ccccccc}
\hline Sex & $\begin{array}{c}\text { Age } \\
\text { (years })\end{array}$ & $\begin{array}{c}\text { BMI } \\
\left(\mathrm{kg} \cdot \mathrm{m}^{-2}\right)\end{array}$ & $\begin{array}{c}\text { Daily steps } \\
\text { at baseline }\end{array}$ & $\begin{array}{c}\text { Change in } \\
\text { daily steps }\end{array}$ & $\begin{array}{c}\text { Number of emails } \\
\text { sent by the participant }\end{array}$ & $\begin{array}{c}\text { Average }(S D) \\
\text { word count of an email }\end{array}$ \\
\hline F & 39 & 33 & 5,327 & -238 & 4 & $121(48)$ \\
M & 43 & 48 & 3,096 & +548 & 4 & $154(81)$ \\
M & 34 & 25 & 7,944 & $+1,794$ & 3 & $79(103)$ \\
M & 64 & 34 & 5,590 & $+3,331$ & 4 & $145(73)$ \\
M & 47 & 28 & 4,289 & $+1,158$ & 2 & $39(11)$ \\
M & 44 & 34 & 5,033 & $+4,592$ & 4 & $58(63)$ \\
M & 30 & 26 & 4,700 & $+2,302$ & 2 & $90(88)$ \\
M & 35 & 38 & 5,582 & $+3,838$ & 5 & $58(38)$ \\
F & 49 & 30 & 5,806 & $+3,958$ & 2 & $57(10)$ \\
F & 51 & 35 & 2,973 & -95 & 1 & $17(\mathrm{~N} / \mathrm{A})$ \\
\hline
\end{tabular}

Note. $\mathrm{BMI}=$ body mass index $; \mathrm{F}=$ female $; \mathrm{M}=$ male $; \mathrm{N} / \mathrm{A}=$ not applicable.

the data. Two researchers repeatedly read the email messages and manually coded the data using pen and paper. The resulting themes were clustered in categories and subcategories. Disagreements were discussed by the two researchers until a consensus was reached.

\section{Ethical considerations}

All participants were informed about all relevant aspects of the study before enrolling, and then provided written informed consent. To ensure their anonymity, potentially identifying features were removed from email messages before analysis. The trial was reviewed and approved by the ethics committee of the Faculty of Physical Education and Sports, Charles University (081/2015), and the study was conducted according to the principles of the Declaration of Helsinki.

\section{Results}

We identified 22 themes that were grouped into 3 categories that were further divided in to a total of 9 subcategories (Table 2).

\section{Reflections on pedometer-based intervention}

In general, the intervention was well accepted by most participants: they enjoyed walking and appreciated the pedometer.

\section{Perceived benefits of PA}

Participants were aware of health benefits of PA and considered walking to be an appropriate and enjoyable form of PA:

"I know that physical activity is very beneficial." (Male, 34)
"I enjoy walking, I'm listening to 'Game of Thrones' while walking, so [I walk] for a long time." (Male, 44)

Walking made them feel better and if they had to interrupt their habitual walking program, they looked forward resuming their walking program:

"... because [walking] makes me really good." (Female, 39)

"After [my present break], I look forward to walking again. I must say, I really miss it." (Female, 39)

\section{Pedometer use}

Most participants appreciated the pedometer and admitted that it was a key element of the intervention:

"I got to like the device." (Male, 35)

"Without the pedometer, I wouldn't have walked that much." (Male, 44)

\section{Use of BCTs}

Action planning, goal setting and self-monitoring were the most popular BCTs implemented by the participants.

Table 2

Key categories and subcategories identified

\begin{tabular}{ll}
\hline Category & Subcategory \\
\hline Reflections on pedometer-based & Perceived benefits of PA \\
intervention & Pedometer use \\
Use of BCTs & Action planning \\
& Goal setting \\
& Self-monitoring \\
& Other BCTs \\
Barriers to engagement in PA & Time constraints \\
& Weather \\
& Lack of motivation \\
\hline
\end{tabular}

Note. $\mathrm{PA}=$ physical activity; $\mathrm{BCTs}=$ behavior change techniques . 


\section{Action planning}

Almost all participants mentioned at least one specific activity they intentionally planned to increase their PA level. Often, they included walking into their commuting routine:

"I try to get off one metro station earlier and walk the rest." (Male, 43)

"I introduced a routine when I go one train stop on foot, which takes approximately 55 minutes." (Male, 44)

Another favorite activity was a leisure walk, sometimes with the company of a dog, which was usually performed in the evening to achieve the daily goal:

"I had to make an evening stroll around the [neighborhood] on purpose, to accomplish my goal." (Female, 39)

"We will try evening and weekend walks with our dogs." (Male, 47)

Other planned activities included intentional increase in work- and family-related errands, and walking up the stairs:

"I started to pick up my kids on foot." (Female, 39)

"In the past, I used to walk up the stairs at work, then I stopped, so I will restart it again." (Male, 43)

Some participants efficiently explored ad hoc opportunities to increase walking:

"After Christmas, I would like to utilize the time when my kids participate in their after-school activities, and walk, at least once a week." (Female, 39)

"I am currently on sick-leave with carpal tendinitis and walk during the slots allowed by my doctor." (Male, 35)

\section{Goal setting}

Most participants accepted the goal set by the researcher and expressed their intention to achieve it:

"By the end of the month, I will definitely get to 10,000." (Male, 44)

"I will try to increase the minimum daily step count gradually every week to at least 8,000." (Male, 35)

Some participants also expressed more or less vague intentions to increase PA levels in the future:

"Starting tomorrow, I will walk again with the pedometer." (Female, 51)

"From January, I will try [walking] again."

(Male, 43)

Two participants had a negative attitude towards their goal: one clearly refused to accept it and the other was very pessimistic with regards to the required behavioral change:

"It is not even realistic to walk 8,000 steps per day."

(Male, 34)

"The increase [in daily step count] will be hard to achieve." (Male, 47)

\section{Self-monitoring}

Generally, participants were aware of their daily steps: "I checked that every evening, and in the last 10 days, I had over 10,000 steps every day; yesterday was even over 15,000.” (Male, 64)

"Every day, my pedometer displays approx. 12,000 steps or more." (Male, 44)

Some participants were able to draw conclusions from their data and tried to predict the impact of their intended activities:

"I can see that during Christmas, I was not doing well." (Female, 39)

"I am curious how the step count increases with an additional walk that lasts approx. 15 minutes." (Male, 43)

For some participants, monitoring their daily steps even became enjoyable:

"Yesterday, during the hiking trip, I enjoyed watching the increasing step [count]." (Male, 35)

"I became enthusiastic about tracking my [step] values and enjoy walking much more.” (Female, 39)

\section{Other BCTS}

Self-reward from achieving the goal was an important factor for two participants:

"On Saturday, I walked 10,000 steps for the first time, and I was really happy about it." (Female, 39)

"I go for a walk just to fulfill the goal of 10,000 steps." (Male, 64)

Another participant built his plan on his past successes, yet another involved his girlfriend to get social support:

"My most successful days are those when I am on a trip to Prague ... mostly in spring and autumn." (Male, 34)

"My girlfriend also got a pedometer." (Male, 47)

Finally, two participants actively pursued habit formation with one of them trying to learn from his previous failures:

"I've become accustomed to regular walks, now it is a natural thing [to go for a walk], especially on weekends." (Male, 64)

"The habit is really important in my case; I failed many times during my effort to lose weight." (Male, 35)

Barrier identification, a BCT itself, has been extensively used by most participants and is discussed in the next section.

\section{Barriers to engagement in PA}

Time constraints, weather conditions, and lack of motivation were the most common barriers that got in the way of increasing PA. 


\section{Time constraints}

Lack of time due to other time-consuming commitments was the most frequent barrier for not achieving goals, with some participants having difficulties to find spare time even on the weekends:

"Due to my job and my daily schedule, it won't be easy to increase my daily number of steps." (Male, 47)

"On the weekend, I need to catch up with my duties from the previous week." (Male, 43)

Long working hours and family commitments were frequently cited:

"I cannot find spare time to walk during my working hours because we are closing the past year ... so I have to sit at the computer." (Female, 39)

"There is a party for fathers at [my child's] kindergarten - I don't want to miss it, and with my working hours, I just won't have time to walk." (Male, 43)

Irregular commitments and unexpected circumstances were also mentioned as barriers on certain days:

"Sometimes I have to drive to work, and in these days, I walk a maximum of 2,000, which decreases my average [daily step count]." (Male, 44)

"I had to stay at home with my sick daughter." (Male, 43)

\section{Weather}

Interestingly, weather was mentioned by 7 participants as a negative factor influencing PA. They often decided not to go for a walk when the weather was too cold and stated that the weather had a large influence on their step count:

"On Friday, it was 13 degrees below zero and I really did not want to go out." (Male, 43)

"The step number is largely dependent on weather - I don't like walking in the cold rain or when it freezes." (Male, 30)

Winter was generally considered as an unfavorable season for walking and participants often expressed expectations that with the upcoming spring, their PA would improve:

"Especially in winter, I don't want to go for a longer walk." (Male, 34)

"I think, with the improving weather, I will improve my physical activity during the weekend thanks to gardening." (Male, 47)

\section{Lack of motivation}

Two participants explained that PA and exercise is not their favorite way of spending time:

"[Physical activities] require extra time that I have to set aside - as such, [this time] is very 'expensive' for me." (Male, 43)

"I do not just aimlessly walk." (Male, 34)

Instead, they preferred mostly sedentary activities:
"As someone considers sitting at the computer or TV as lost time, with me it is quite opposite - we are all different." (Male, 43)

"In my free time, I like to lie around, watch TV, and do nothing." (Male, 34)

\section{Discussion}

By thematically analyzing the email messages written by primary care patients participating in an email counseling intervention meant to increase PA, we have shown how patients implicitly use various BCTs in their efforts to increase PA levels. While some of the BCTs were actively prompted as part of the counseling support (action planning, goal-setting, self-monitoring), others were brought up by the patients themselves (selfreward, habit formation, social support). Additionally, we have identified several barriers that they encountered while doing so.

\section{Results in the context of other literature}

The field of BCTs is very well researched (Michie et al., 2011, 2013) and BCTs are widely used to develop, implement, and report health behavior interventions, including PA interventions (Bird et al., 2013; Cradock et al., 2017; Olander et al., 2013). Nevertheless, little is known about how the participants in these interventions actually perceive and use these BCTs (Casey et al., 2014; Normansell et al., 2014).

Normansell et al. interviewed a sample of 43 participants from the PACE-UP trial, a randomized controlled trial aiming to increase walking in 45-75 years old inactive primary care patients through the use of pedometers with and without additional support from a practice nurse (Harris et al., 2017). They found 152 discrete examples of different BCTs being alluded to by participants and attempted to assign them to one of the BCTs specified in the trial protocol, for example: reviewing goals and outcomes, providing feedback, planning social support, and prompting self-monitoring (Normansell et al., 2014). Self-monitoring is a common BCT, as it was also found in our study in addition to another study using 12 participants from the SMART MOVE trial, an open-label randomized controlled trial of a smartphone app that was meant to promote PA in primary care (Glynn et al., 2014). While the authors did not specifically attempt to systematically identify BCTs, several other themes emerged from their analysis that can be considered as BCTs such as goal setting, feedback, and rewards (Casey et al., 2014).

Our data supplements these prior findings by documenting several BCTs that had not been mentioned in either of the previous two papers (Casey et al., 2014; 
Normansell et al., 2014). Of note is action planning, which has been alluded to by almost all of our participants as they listed various activities, they consciously planned to increase their PA levels (walking as part of commuting or family-related errands, walking a dog).

Furthermore, we have anecdotally described several interesting aspects of other BCTs. Firstly, establishing goals and reminding the participants of their goals was a BCT mentioned in both previously published papers (Casey et al., 2014; Normansell et al., 2014), but neither of them reported the negative attitude elicited by the set goal. The prevalence of this negative attitude and its impact on the counseling process need to be quantified in future studies to determine its relevance in the counseling practice. Additionally, we have described how participants learn from their data. This technique, related to self-monitoring, should be further investigated to see whether actively prompting participants to learn from their own data improves counseling outcomes, and if so, what are the best ways to facilitate their learning process. Finally, we have noticed that some participants reported enjoyment related to self-monitoring with pedometers. This phenomenon has been reported previously (De Cocker, De Bourdeaudhuij, \& Cardon, 2008) and future studies should explore whether it is associated with increases in PA levels and eventually how enhancing enjoyment improves the effectiveness of PA interventions.

As for the barriers to PA, this study is in agreement with prior work, confirming that lack of time and unfavorable weather conditions are the most common barriers for increasing and maintaining PA (Brawley, Rejeski, \& King, 2003; Kelly et al., 2016; Normansell et al., 2014; Trost, Owen, Bauman, Sallis, \& Brown, 2002; Wahlich et al., 2017). In addition, two participants explicitly expressed their preference of sedentary lifestyle and lack of motivation as a barrier to increase their PA levels. Though it might be a coincidence, it is of note that both of them were middle-aged men, which is in line with previous research demonstrating that this age group commonly report laziness to be physically active, low appeal of PA programs, and decreased motivation for PA with increasing age (Brunet \& Sabiston, 2011; Caperchione et al., 2012).

\section{Study strengths and limitations}

The strength of this study is the unique qualitative approach using the thematic analysis of the email messages written as responses to a counselor. This context diminishes the risk that participants provide justifications of their actions instead of explanations for them, which can happen in interviews, especially when interviewees are asked about contentious or emotional topics (Diefenbach, 2008; Salmon, 2013).
Furthermore, the possibility to analyze a longitudinal sample of multiple email messages from each participant spread throughout the 12-week intervention was an opportunity to explore participants' coping with bad weather and work-related lack of time. Specifically, some participants expressed expectations to increase their daily steps during the upcoming spring season or with a change in their job responsibilities, however, these expectations often remained unfulfilled.

Our method of analyzing the email messages is not without its own limitations when compared to traditional interviews. For example, the email communication lacks verbal and nonverbal cues which limits its ability to convey important information (Pelling, 2009). Additionally, writing emails requires a certain level of computer literacy, which makes it unsuitable for some populations, for example older adults. Indeed, the oldest of our participants (Male, 64) did not use email and asked his daughter to write the emails on his behalf.

The obvious limitation of our study is the low number and heterogeneity of participants, whose emails could be analyzed. Specifically, the substantial heterogeneity of the participants with respect to their baseline levels of physical activity (ranging from 3,100 to 7,900 steps per day), body mass index (range 25 to $48 \mathrm{~kg} \cdot \mathrm{m}^{-2}$ ), and age (range 30 to 64 years) limits the applicability of the study findings to particular individuals (e.g., young obese vs older active). Furthermore, the low number of participants did not allow us to reach sufficient saturation of themes as others have recommended (Finset, 2008). Despite our inability to reach saturation, we described several themes that were not reported in prior work based on interviews with 43 participants that reached saturation (Normansell et al., 2014). This might indicate that thematic analysis of email messages can yield additional insights that are difficult to reveal otherwise and is thus suitable as a complementary method to post-intervention interviews.

\section{Conclusion}

Using thematic analysis of the email messages written by participants in the course of physical activity counseling intervention, the study extends previous findings on the use of BCTs by intervention participants, documenting their perceptions and experiences with various BCTs. The study also identifies common barriers encountered by intervention participants in their effort to increase their level of PA.

Understanding how the participants of PA interventions perceive and use various BCTs that form the basis of the intervention is crucial for researchers and 
clinicians alike. This study shows that BCTs like action planning, self-monitoring, goal setting, and barrier identification can be widely adopted by intervention participants. Additionally, this study describes several interesting aspects of these BCTs that should be taken into consideration when designing an intervention (e.g., negative attitudes to goal setting, participants learning from their own data, self-monitoring as enjoyable activity). Finally, the study documents participants' experiences of various barriers to PA and thus can be helpful in developing strategies to overcome these barriers.

\section{Acknowledgment}

The study was funded in part by a research program of Charles University (Progres Q41).

\section{Conflict of interest}

There were no conflicts of interest.

\section{References}

Bardach, S. H., \& Schoenberg, N. E. (2014). The content of diet and physical activity consultations with older adults in primary care. Patient Education and Counseling, 95, 319-324.

Bird, E. L., Baker, G., Mutrie, N., Ogilvie, D., Sahlqvist, S., \& Powell, J. (2013). Behavior change techniques used to promote walking and cycling: A systematic review. Health Psychology, 32, 829-838.

Brawley, L. R., Rejeski, W. J., \& King, A. C. (2003). Promoting physical activity for older adults: The challenges for changing behavior. American Journal of Preventive Medicine, 25(3 Suppl. 2), 172-183.

Brunet, J., \& Sabiston, C. M. (2011). Exploring motivation for physical activity across the adult lifespan. Psychology of Sport and Exercise, 12, 99-105.

Caperchione, C. M., Vandelanotte, C., Kolt, G. S., Duncan, M., Ellison, M., George, E., \& Mummery, W. K. (2012). What a man wants: Understanding the challenges and motivations to physical activity participation and healthy eating in middle-aged Australian men. American Journal of Men's Health, 6, 453-461.

Carroll, J. K., Antognoli, E., \& Flocke, S. A. (2011). Evaluation of physical activity counseling in primary care using direct observation of the 5As. Annals of Family Medicine, 9, 416-422.

Casey, M., Hayes, P. S., Glynn, F., Ólaighin, G., Heaney, D., Murphy, A. W., \& Glynn, L. G. (2014). Patients' experiences of using a smartphone application to increase physical activity: The SMART MOVE qualitative study in primary care. British Journal of General Practice, 64, e500-e508.
Chiu, T. M. L., Marziali, E., Tang, M., Colantonio, A., \& Carswell, A. (2011). Client-centred concepts in a personalized e-mail support intervention designed for Chinese caregivers of family members with dementia: A qualitative study. Hong Kong Journal of Occupational Therapy, 20, 87-93.

Cradock, K. A., Ólaighin, G., Finucane, F. M., Gainforth, H. L., Quinlan, L. R., \& Ginis, K. A. M. (2017). Behaviour change techniques targeting both diet and physical activity in type 2 diabetes: A systematic review and meta-analysis. International Journal of Behavioral Nutrition and Physical Activity, 14, 18-35.

De Cocker, K. A., De Bourdeaudhuij, I. M., \& Cardon, G. M. (2008). The effect of pedometer use in combination with cognitive and behavioral support materials to promote physical activity. Patient Education and Counseling, 70, 209-214.

Diefenbach, T. (2008). Are case studies more than sophisticated storytelling? Methodological problems of qualitative empirical research mainly based on semi-structured interviews. Quality \& Quantity, 43, 875-894.

Egan, J., Chenoweth, L., \& McAuliffe, D. (2009). Emailfacilitated qualitative interviews with traumatic brain injury survivors: A new and accessible method. Brain Injury, 20, 1283-1294.

Finset, A. (2008). Qualitative methods in communication and patient education research. Patient Education and Counseling, 73, 1-2.

Gagliardi, A. R., Abdallah, F., Faulkner, G., Ciliska, D., \& Hicks, A. (2015). Factors contributing to the effectiveness of physical activity counselling in primary care: A realist systematic review. Patient Education and Counseling, 98, 412-419.

Gao, S., Stone, R. A., Hough, L. J., Haibach, J. P., Marcus, B. H., Ciccolo, J. T., ... Sevick, M. A. (2016). Physical activity counseling in overweight and obese primary care patients: Outcomes of the VA-STRIDE randomized controlled trial. Preventive Medicine Reports, 3, 113-120.

Glynn, L. G., Hayes, P. S., Casey, M., Glynn, F., AlvarezIglesias, A., Newell, J., ... Murphy, A. W. (2014). Effectiveness of a smartphone application to promote physical activity in primary care: The SMART MOVE randomised controlled trial. British Journal of General Practice, 64, e384-e391.

Hageman, P. A., Pullen, C. H., Hertzog, M., Pozehl, B., Eisenhauer, C., \& Boeckner, L. S. (2017). Web-based interventions alone or supplemented with peer-led support or professional email counseling for weight loss and weight maintenance in women from rural communities: Results of a clinical trial. Journal of Obesity, 2017, 1602627.

Harris, T., Kerry, S. M., Limb, E. S., Victor, C. R., Iliffe, S., Ussher, M., ... Cook, D. G. (2017). Effect of a primary care walking intervention with and without nurse support on physical activity levels in 45- to 75-year-olds: The pedometer and consultation evaluation (PACE-UP) cluster randomised clinical trial. PLOS Medicine, 14, e1002210.

Harris, T., Kerry, S. M., Victor, C. R., Ekelund, U., Woodcock, A., Iliffe, S., ... Cook, D. G. (2015). A primary care nurse-delivered walking intervention in older adults: PACE (Pedometer Accelerometer Consultation Evaluation)-Lift cluster randomised controlled trial. PLOS Medicine, 12, e1001783. 
Hatchett, A., Hallam, J. S., \& Ford, M. A. (2012). Evaluation of a social cognitive theory-based email intervention designed to influence the physical activity of survivors of breast cancer. Psycho-Oncology, 22, 829-836.

Hébert, E. T., Caughy, M. O., \& Shuval, K. (2012). Primary care providers' perceptions of physical activity counselling in a clinical setting: A systematic review. British Journal of Sports Medicine, 46, 625-631.

Hershberger, P. E., \& Kavanaugh, K. (2017). Comparing appropriateness and equivalence of email interviews to phone interviews in qualitative research on reproductive decisions. Applied Nursing Research, 37, 50-54.

James, E. L., Ewald, B. D., Johnson, N. A., Stacey, F. G., Brown, W. J., Holliday, E. G., ... Plotnikoff, R. C. (2017). Referral for expert physical activity counseling: A pragmatic RCT. American Journal of Preventive Medicine, 53, 490-499.

Jimmy, G., \& Martin, B. W. (2005). Implementation and effectiveness of a primary care based physical activity counselling scheme. Patient Education and Counseling, 56, 323-331.

Kelly, S., Martin, S., Kuhn, I., Cowan, A., Brayne, C., \& Lafortune, L. (2016). Barriers and facilitators to the uptake and maintenance of healthy behaviours by people at mid-life: A rapid systematic review. PLOS ONE, 11, e0145074.

Michie, S., Ashford, S., Sniehotta, F. F., Dombrowski, S. U., Bishop, A., \& French, D. P. (2011). A refined taxonomy of behaviour change techniques to help people change their physical activity and healthy eating behaviours: The CALO-RE taxonomy. Psychology \& Health, 26, 1479-1498.

Michie, S., Richardson, M., Johnston, M., Abraham, C., Francis, J., Hardeman, W., ... Wood, C. E. (2013). The behavior change technique taxonomy (v1) of 93 hierarchically clustered techniques: Building an international consensus for the reporting of behavior change interventions. Annals of Behavioral Medicine, 46, 81-95.

Normansell, R., Smith, J., Victor, C., Cook, D. G., Kerry, S., Iliffe, S., ... Harris, T. (2014). Numbers are not the whole story: A qualitative exploration of barriers and facilitators to increased physical activity in a primary care based walking intervention. BMC Public Health, 14, 1272.

Olander, E. K., Fletcher, H., Williams, S., Atkinson, L., Turner, A., \& French, D. P. (2013). What are the most effective techniques in changing obese individuals' physical activity self-efficacy and behaviour: A systematic review and meta-analysis. International Journal of Behavioral Nutrition and Physical Activity, 10, 29.

Orrow, G., Kinmonth, A.-L., Sanderson, S., \& Sutton, S. (2012). Effectiveness of physical activity promotion based in primary care: Systematic review and meta-analysis of randomised controlled trials. BMJ, 344, e1389.

Patel, A., Schofield, G. M., Kolt, G. S., \& Keogh, J. W. L. (2013). Perceived barriers, benefits, and motives for physical activity: Two primary-care physical activity prescription programs. Journal of Aging and Physical Activity, 21, 85-99.
Pears, S., Bijker, M., Morton, K., Vasconcelos, J., Parker, R. A., Westgate, K., ... Hardeman, W. (2016). A randomised controlled trial of three very brief interventions for physical activity in primary care. BMC Public Health, 16, 1033.

Pedersen, B. K., \& Saltin, B. (2015). Exercise as medicine - evidence for prescribing exercise as therapy in 26 different chronic diseases. Scandinavian Journal of Medicine \& Science in Sports, 25, 1-72.

Pelling, N. J. (2009). The use of email and the Internet in counselling and psychological service: What practitioners need to know. Counselling, Psychotherapy, and Health, 5, $1-25$.

Reiner, M., Niermann, C., Jekauc, D., \& Woll, A. (2013). Long-term health benefits of physical activity - a systematic review of longitudinal studies. BMC Public Health, 13, 813.

Richards, E. A., \& Cai, Y. (2016). Integrative review of nurse-delivered physical activity interventions in primary care. Western Journal of Nursing Research, 38, 484-507.

Salmon, P. (2013). Assessing the quality of qualitative research. Patient Education and Counseling, 90, 1-3.

Trost, S. G., Owen, N., Bauman, A. E., Sallis, J. F., \& Brown, W. (2002). Correlates of adults' participation in physical activity: Review and update. Medicine \& Science in Sports \& Exercise, 34, 1996-2001.

van der Weegen, S., Verwey, R., Spreeuwenberg, M., Tange, H., van der Weijden, T., \& de Witte, L. (2015). It's LiFe! Mobile and web-based monitoring and feedback tool embedded in primary care increases physical activity: A cluster randomized controlled trial. Journal of Medical Internet Research, 17, e184.

van Esch, S. C., Cornel, M. C., \& Snoek, F. J. (2010). “I am pregnant and my husband has diabetes. Is there a risk for my child?" A qualitative study of questions asked by email about the role of genetic susceptibility to diabetes. BMC Public Health, 10, 67-68.

Vetrovsky, T., Cupka, J., Dudek, M., Kuthanova, B., Vetrovska, K., Capek, V., \& Bunc, V. (2018). A pedometer-based walking intervention with and without email counseling in general practice: A pilot randomized controlled trial. $B M C$ Public Health, 18, 635-647.

Wahlich, C., Beighton, C., Victor, C., Normansell, R., Cook, D., Kerry, S., ... Harris, T. (2017). "You started something ... then I continued by myself": A qualitative study of physical activity maintenance. Primary Health Care Research \& Development, 1, 1-17.

Warburton, D. E. R., Nicol, C. W., \& Bredin, S. S. D. (2006). Health benefits of physical activity: The evidence. Canadian Medical Association Journal, 174, 801-809.

Woodcock, J., Franco, O. H., Orsini, N., \& Roberts, I. (2011). Non-vigorous physical activity and all-cause mortality: Systematic review and meta-analysis of cohort studies. International Journal of Epidemiology, 40, 121-138.

Yates, T., Edwardson, C. L., Henson, J., Gray, L. J., Ashra, N. B., Troughton, J., ... Davies, M. J. (2017). Walking away from type 2 diabetes: A cluster randomized controlled trial. Diabetic Medicine, 34, 698-707. 